\title{
Presencia de hongos filamentosos en yerba mate compuesta y eficiencia de medios de cultivo para el aislamiento de Aspergillus
}

\author{
Presence of filamentous fungi in composite yerba mate and efficiency of culture \\ media for isolation of Aspergillus
}

\author{
Andrea Alejandra Arrúa Alvarenga ${ }^{1 *}$, Inocencia Palmira Peralta Lòpez ${ }^{2}$, Cinthia Mabel \\ Rojas Abraham $^{3}$, Yessica Magaliz Reyes Caballero ${ }^{3}$, Carolina Toledo Popoff ${ }^{3}$, Leticia \\ Vazquez $^{4}$, y Juliana Moura Mendes Arrua ${ }^{1}$
}

\footnotetext{
${ }^{1}$ Departamento de Biotecnología, Centro Multidisciplinario de Investigaciones Tecnológicas, Dirección General de Investigación Científica y Tecnológica, Universidad Nacional de Asunción (CEMIT, DGICT, UNA). Campus Universitario, San Lorenzo, Paraguay.

${ }^{2}$ DGICT, UNA. Campus Universitario, San Lorenzo, Paraguay.

${ }^{3}$ Iniciación científica, Departamento de Biotecnología, CEMIT, DGICT, UNA. Campus Universitario, San Lorenzo, Paraguay.

Autor para correspondencia (aaarrua@gmail.com)

Recibido: 10/02/2016; Aceptado: 16/06/2016.

Doi:10.18004/investig.agrar.2016.junio.49-55
}

\section{RESUMEN}

La yerba mate es una planta originaria de Sudamérica y de consumo masivo en Paraguay. La yerba compuesta, aquella con agregado de hierbas medicinales, es de alto consumo y aceptación en el mercado. Durante su cultivo y procesamiento los componentes de la yerba compuesta se ven expuestos al ataque de hongos, entre ellos los pertenecientes al género Aspergillus spp., potencialmente productor de micotoxinas dañinas para el ser humano. Con el objetivo de determinar el medio de cultivo más eficiente para aislar hongos del género Aspergillus spp., se compararon cinco diferentes preparaciones. Se identificaron diez géneros de hongos, y dentro del género Aspergillus spp., cuatro Secciones: Flavi, Nigri, Circumdati y Fumigati. Se concluyó que el Medio Específico para Aislamiento de Aspergillus MAAS, fue el más eficaz para este fin.

Palabras clave: hongos, Ilex paraguariensis, micotoxinas.

\begin{abstract}
Yerba mate is a plant native to South America and high consumption in Paraguay. The composite yerba mate, with added herbs is high consumption and market acceptance. During its cultivation and processing, components of the composite yerba are exposed to fungal attack, including those belonging to the genus Aspergillus spp, producing potentially harmful mycotoxins for humans. In order to determine the most efficient medium for isolating fungi Aspergillus spp., five different formulations were compared. Ten genera of fungi were identified and within the genus Aspergillus four sections: Flavi, Nigri, Circumdati and Fumigati. It was concluded that specific media for isolating Aspergillus MAAS, was the most efficient for this purpose.

Key words: fungi, Ilex paraguariensis, mycotoxins.
\end{abstract}




\section{INTRODUCCIÓN}

La yerba mate, Ilex paraguariensis es una planta originaria de Sudamérica, tradicionalmente utilizada por los indígenas que habitaban las regiones de Paraguay, nordeste de Argentina y norte de Uruguay (Pérez Paiva et al. 2012).

Actualmente su consumo se ha difundido y es utilizada en lugares como Estados Unidos, Europa y Asia (Pérez Paiva et al. 2012). En Paraguay, su consumo se halla ampliamente difundido en formas variadas como mate frío o terere, mate caliente y cocido (De Bernardi y Prat 2001, Pérez Paiva et al. 2012. La demanda del mercado interno ha aumentado aproximadamente $20 \%$ en los últimos años (REDIEX 2015).

La yerba mate compuesta, es un producto derivado de la yerba mate, con el agregado de otras hierbas medicinales que confieren un sabor y propiedades diferentes a la yerba mate tradicional y que actualmente posee gran aceptación por parte de los consumidores (Pérez Paiva et al. 2012).

Durante su cultivo y almacenamiento, los productos agrícolas se ven expuestos al ataque de microorganismos, entre ellos los hongos. Si las condiciones de temperatura y almacenamiento son adecuadas se favorece el desarrollo de estos hongos, entre ellos los productores de micotoxinas, metabolitos secundarios tóxicos para los seres humanos y animales que producen síndromes llamados micotoxicosis y que en casos extremos pueden llevar a la muerte (Soriano 2007).

A pesar de la abundancia en la naturaleza de los hongos potencialmente productores de micotoxinas, especialmente aquellos pertenecientes al género Aspergillus y cuya presencia en los alimentos ha sido estudiada de larga data, su aislamiento de sustratos alimentarios y su identificación puede resultar compleja.

La sistemática de Aspergillus se ha visto influida en los últimos años por trabajos presentados en reuniones científicas dedicadas exclusivamente a los géneros Penicillium y Aspergillus. El género Aspergillus se divide en seis subgéneros, divididos a su vez en una o dos secciones (Gams et al. 1985, Abarca 2000).

Los criterios seguidos actualmente para clasificar las especies del género Aspergillus y sus teleomorfos son principalmente morfológicos, tanto macro como micro morfológicos; no obstante, en algunas secciones se han realizado además estudios bioquímicos o moleculares encaminados a resolver algunos de los problemas planteados en su clasificación, esto se conoce como identificación polifásica (Abarca 2000, Samson et al. 2014).

El sistema de identificación propuesto por Klich y Pitt, utiliza tres medios de cultivo y dos temperaturas de incubación, 25 y $37^{\circ} \mathrm{C}$. Tras siete días de incubación se procede a la observación de las características morfológicas macroscópicas y microscópicas de los cultivos (Klich y Pitt 1988, Klich 2002). Dentro de las diferentes secciones en que el género se subdivide se encuentran especies tipo que son importantes productoras de micotoxinas. En la Sección Flavi, la especie tipo Aspergillus flavus ha sido ampliamente documentada como la más importante productora de aflatoxinas; en la Sección Nigri, la especie tipo, Aspergillus niger se cita como la mayor productora de ocratoxinas; en cuanto a la Sección Circumdati, Aspergillus ochraceus es también citado como productor de ocratoxinas (Abarca 2000, Samson et al. 2014).

Se ha reportado la presencia de hongos de los géneros Aspergillus niger, Aspergillus flavus y Aspergillus ochraceus en yerba mate elaborada en el país (Pérez Paiva et al. 2012). Aspergillus niger es el más importante productor de ocratoxinas, junto con Aspergillus ochraceus. Aspergillus flavus es productor de aflatoxinas (Soriano 2007).

Las ocratoxinas son neurotóxicas, teratogénicas e inmunotóxicas (López de Cerain y Soriano 2007, Bezerra da Rocha et al. 2014, Wu et al. 2014; BuiKlimke y Wu 2015). Las aflatoxinas producen daño hepático agudo, cirrosis, son oncogénicas, teratogénicas, inmunotóxicas, se acumulan en los tejidos y se excretan por la leche (Juan et al. 2007, Bezerra da Rocha et al. 2014, Wu et al. 2014).

El consumo de yerba mate compuesta contaminada con hongos aflatoxigénicos y ocratoxigénicos representa un riesgo potencial para la salud pública puesto que la exposición a altos niveles de aflatoxinas y ocratoxinas puede producir daño a la salud de la población, especialmente en un país como Paraguay donde este producto se consume de manera masiva (Pérez Paiva et al. 2012).

La inocuidad alimentaria es un tema relevante, puesto que no solo se debe ofrecer a los consumidores alimentos de calidad, sino inocuos y que no produzcan daños posteriores a su salud, por tanto es de suma importancia 
determinar el medio más adecuado para la captura y aislamiento de hongos del género Aspergillus spp. provenientes de yerba mate. No existen trabajos previos al respecto y por lo general se utilizan medios generales para conteo de hongos y levaduras que podrían no ser eficientes para que los Aspergillus spp. crezcan o bien podrían quedar enmascarados debido a la presencia de otros organismos que crecen de manera más rápida o utilizan los nutrientes de los medios generales de manera más eficiente, por tanto, los objetivos de este trabajo fueron, identificar los géneros de hongos filamentosos presentes en muestras de yerba mate compuesta y evaluar la eficiencia de diferentes medios de cultivo para el aislamiento de hongos del género Aspergillus spp. presentes en yerba mate compuesta.

\section{MATERIALES Y MÉTODOS}

El trabajo se llevó a cabo en el Laboratorio de Biotecnología del Centro Multidisciplinario de Investigaciones Tecnológicas, CEMIT-DGICT-UNA, durante el mes de enero del año 2016. Se utilizó un diseño observacional descriptivo de corte transversal. Fueron incluidas tres (3) marcas de yerba mate compuesta comercial con hierbas medicinales y cinco diferentes medios de cultivo: 1. Papa dextrosa agar (PDA) (200 g de papa natural, $20 \mathrm{~g}$ de dextrosa anhidra, $20 \mathrm{~g}$ de agar $\mathrm{y}$ $1000 \mathrm{~mL}$ de agua destilada) 2. Extracto de malta agar al 6\% (MSA) (20 g de extracto de malta, $20 \mathrm{~g}$ de agar bacteriológico, $60 \mathrm{~g}$ de cloruro de sodio y $1000 \mathrm{~mL}$ de agua destilada), 3. Agar agua (AA) (20 g de agar agar y $1000 \mathrm{~mL}$ de agua destilada), 4. Czapek (CZ) (2 g de nitrato sódico, $0,5 \mathrm{~g}$ de cloruro potásico, $0,5 \mathrm{~g}$ de sulfato magnésico, 0,01 $\mathrm{g}$ de sulfato ferroso, $1 \mathrm{~g}$ de fosfato di potásico, $30 \mathrm{~g}$ de sacarosa, $15 \mathrm{~g}$ de agar agar y $1000 \mathrm{~mL}$ de agua destilada) y Medio selectivo para aislamiento de Aspergillus (MAAS) (de suelo) (5 $\mathrm{g}$ de peptona, $1 \mathrm{~g}$ de hipofosfito de potasio, $30 \mathrm{~g}$ de cloruro de sodio, $10 \mathrm{~g}$ de glucosa, 0,5 $\mathrm{g}$ de sulfato de magnesio heptahidratado, 20 g de agar y $1000 \mathrm{~mL}$ de agua destilada) (Dhingra y Sinclair 1995, Klich 2002, Cabañes et al. 2007).

Se realizó una dilución de $1 / 10$ de la yerba mate compuesta en agua destilada esterilizada y se sembraron $10 \mathrm{~mL}$ por triplicado en placas de Petri de vidrio de $9 \mathrm{~cm}$ de diámetro, en el centro de la placa y se distribuyó con un ansa de Digralsky. Posteriormente las placas se incubaron a $25 \pm 2{ }^{\circ} \mathrm{C}$. Transcurridas $24 \mathrm{~h}$ se voltearon para facilitar el desarrollo de las colonias y se incubaron por $48 \mathrm{~h}$ más a $25 \pm 2^{\circ} \mathrm{C}$.
Una vez crecidas las colonias, las mismas fueron purificadas en medio PDA e identificadas mediante el uso de claves taxonómicas y bases de datos online (Barnett y Hunter 1986, Robert et al. 2013).

Los hongos pertenecientes al género Aspergillus spp. fueron purificados en PDA y se realizó su identificación a nivel de Sección mediante el uso de claves (Klich 2002).

Se calculó incidencia de los hongos presentes como porcentaje como unidades formadoras de colonias. El perfil micológico se evaluó mediante el conteo de colonias de los géneros de hongos presentes en las muestras estudiadas. Posteriormente se realizó la prueba de normalidad de Shapiro-Wilk (Shapiro y Wilk 1965) modificada para cada tipo de muestra estudiada. Para la comparación de medias se utilizó el análisis de varianza no paramétrica de Kruskal-Wallis (Kruskal y Wallis 1952) con un intervalo de confianza del $95 \%$, por medio del paquete estadístico InfoStat(Di Rienzo et al. 2011).

\section{RESULTADOS Y DISCUSION}

Fueron aislados hongos de los géneros Acremonium sp., Aspergillus spp., Cladosporium sp., Chalara sp., Fusarium spp., Nigrospora sp. Paecilomyces sp., Penicillium spp., Torula sp. y Trichoderma sp. Cabe destacar que es la primera vez que se determina la presencia de Acremonium sp., Cladosporium sp., Chalara sp., Nigrospora sp., Paecilomyces sp., Torula sp. y Trichoderma sp. En los medios de cultivo estudiados las incidencias de hongos presentes fueron variables debido posiblemente a diferencias entre los productos comerciales que podrían deberse a su manejo durante el proceso de elaboración de la yerba compuesta y condiciones de almacenamiento posterior y la constitución nutricional de los medios utilizados para este estudio (Tabla 1).

Estos resultados demuestran que la yerba mate es un sustrato adecuado para el desarrollo de diferentes especies de hongos, incluyendo al género Aspergillus spp., por tanto es fundamental determinar el medio más adecuado para la captura de estos hongos y que pueda indicar realmente la incidencia de los mismos en yerba mate, dado su potencial riesgo a la salud humana.

Existen pocos trabajos realizados sobre las especies de hongos presentes en yerba mate elaborada. Por lo general, los aislamientos de hongos se realizan en medios recomendados para el conteo en general de hongos y 
levaduras y establecidos en normas nacionales e 2001, RDC ANVISA / Nro. 302 2002, IRAM 2004) internacionales (INTN 1995, RDC ANVISA/ Nro. 12

Tabla 1. Porcentaje de incidencia de géneros de hongos en yerba mate compuesta en agar agua (AA), papa dextrosa agar (PDA), czapek (CZ), malta sal agar 6\% (MSA) y medio selectivo para aislamiento de Aspergillus (MAAS).

\begin{tabular}{|c|c|c|c|c|c|c|c|c|c|c|c|}
\hline Yerba compuesta & 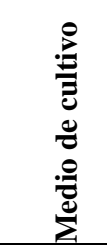 & 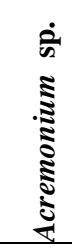 & 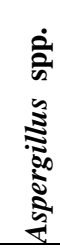 & 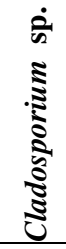 & 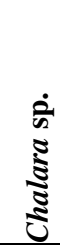 & 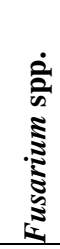 & 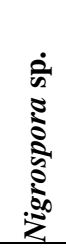 & 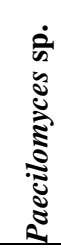 & 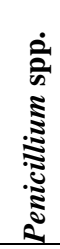 & 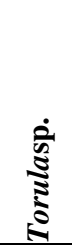 & 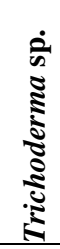 \\
\hline \multirow{5}{*}{ Marca A } & AA & $10 *$ & 10 & 0 & 0 & 10 & 20 & 0 & 30 & 0 & 20 \\
\hline & PDA & 0 & 30 & 30 & 0 & 0 & 0 & 0 & 10 & 30 & 0 \\
\hline & $\mathrm{CZ}$ & 0 & 10 & 10 & 20 & 0 & 0 & 0 & 20 & 10 & 30 \\
\hline & MSA & 0 & 30 & 30 & 0 & 0 & 0 & 0 & 20 & 0 & 20 \\
\hline & MAAS & 0 & 30 & 20 & 0 & 0 & 0 & 0 & 20 & 10 & 20 \\
\hline \multirow{5}{*}{ Marca B } & $\mathrm{AA}$ & 10 & 20 & 30 & 10 & 0 & 20 & 10 & 0 & 0 & 0 \\
\hline & PDA & 0 & 10 & 10 & 0 & 0 & 0 & 0 & 30 & 0 & 50 \\
\hline & $\mathrm{CZ}$ & 0 & 30 & 10 & 0 & 10 & 0 & 0 & 20 & 0 & 30 \\
\hline & MSA & 0 & 20 & 30 & 0 & 0 & 0 & 0 & 40 & 0 & 10 \\
\hline & MAAS & 0 & 20 & 20 & 0 & 0 & 0 & 0 & 30 & 0 & 30 \\
\hline \multirow{5}{*}{ Marca C } & AA & 10 & 10 & 10 & 0 & 0 & 10 & 0 & 30 & 10 & 20 \\
\hline & PDA & 10 & 10 & 10 & 0 & 0 & 0 & 10 & 40 & 0 & 20 \\
\hline & $\mathrm{CZ}$ & 0 & 20 & 30 & 0 & 0 & 0 & 0 & 30 & 0 & 20 \\
\hline & MSA & 0 & 10 & 30 & 0 & 0 & 20 & 0 & 30 & 0 & 10 \\
\hline & MAAS & 0 & 40 & 0 & 10 & 0 & 0 & 10 & 20 & 0 & 20 \\
\hline
\end{tabular}

*Valores expresados como porcentaje de hongos presentes en las muestras analizadas.

Los géneros identificados coinciden con los reportados por Pérez Paiva et al. (2012) que observaron la presencia de Aspergillus spp. y Fusarium spp. en muestras de yerba mate compuesta, empleando el método de siembra en profundidad en agar oxitetraciclina de acuerdo con la metodología propuesta en ICMSF (The International Commission on Microbiological Specificatications for Foods). También coincide con lo encontrado por González et al. (2012) que determinaron la presencia de los géneros Cladosporium sp., Aspergillus spp., Fusarium spp., y Penicillium spp. entre otros en yerba mate compuesta. No puede asegurarse la procedencia de los hongos identificados puesto que podrían ser contaminantes de la yerba mate o bien de las hierbas medicinales utilizadas en la elaboración de la yerba compuesta.

Jerke et al. (2009), determinaron la presencia de 24 géneros de hongos filamentosos en muestras de yerba mate elaborada, empleando el método de diseminación en superficie en agar-cloranfenicol de acuerdo con la metodología propuesta en la Norma IRAM 20517:2004 (IRAM 2004, IRAM 2007). Dentro de los géneros de mohos micotoxigénicos el mayor porcentaje de incidencia le correspondió al género Aspergillus spp. (86\%), seguido de Penicillium spp. (26\%) y Fusarium spp. (15\%).

En cuanto a los Aspergillus spp. en AA, las incidencias se mantuvieron en $10 \%$, en PDA entre 10 y $30 \%$, en $\mathrm{CZ}$ entre 10 y $30 \%$, en MSA entre 10 y $30 \%$ y en MAAS entre 20 y $40 \%$. El MAAS presentó las incidencias más altas de hongos del género Aspergillus spp. en comparación con los demás medios estudiados.

Al realizar el análisis estadístico se observaron diferencias significativas en referencia a la incidencia de Aspergillus spp. en los diferentes medios estudiados. El medio MAAS, fue el más eficiente para el aislamiento de estos hongos, a pesar de que este medio está recomendado para aislar Aspergillus del suelo, fue eficaz para capturar hongos de yerba mate (Figura 1). 


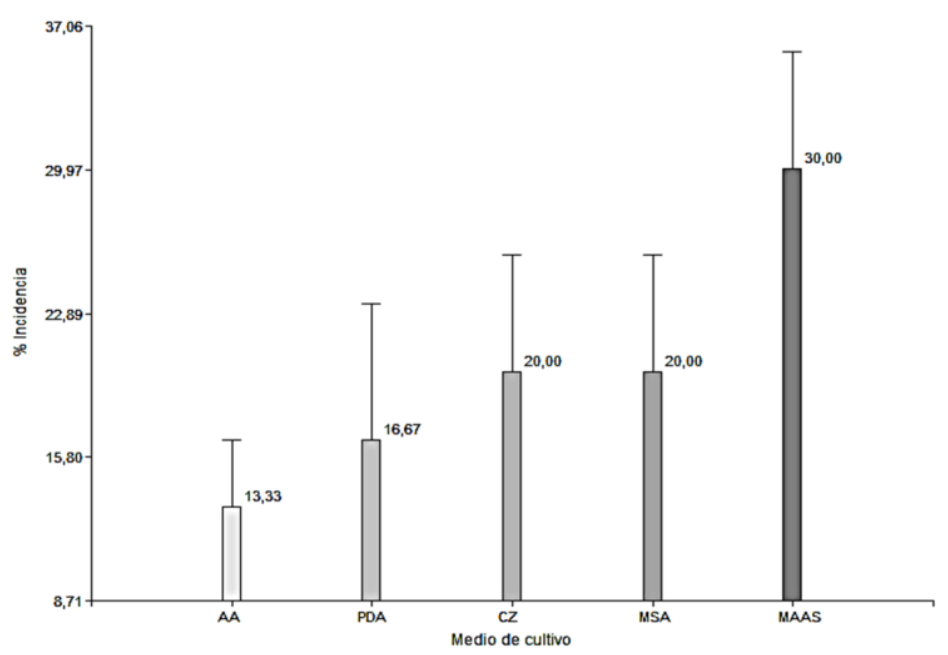

Figura 1. Incidencia de Aspergillus spp. en los diferentes medios de cultivo estudiados. Agar agua (AA), papa dextrosa agar (PDA), czapek (CZ), malta sal agar 6\% (MSA) y medio selectivo para aislamiento de Aspergillus (MAAS).

Los diferentes géneros de hongos filamentosos poseen necesidades nutricionales y de agua que difieren entre los mismos y dentro de un género entre especies, por tanto, la composición del medio podría influenciar el crecimiento y desarrollo de los organismos (Sanchis et al. 2007, Giorni 2011).
La presencia de hongos saprofitos se podría considerar como indicador del grado de degradación de las muestras y las condiciones de almacenamiento de las mismas.

Las secciones de Aspergillus spp. presentes en los diferentes medios de cultivo y posteriormente purificadas e identificadas se presentan en la Tabla 2.

Tabla 2. Porcentaje de incidencia de Aspergillus spp. en yerba mate compuesta en agar agua (AA), papa dextrosa agar (PDA), czapek (CZ), malta sal agar 6\% (MSA) y medio selectivo para aislamiento de Aspergillus spp. (MAAS).

\begin{tabular}{clcccc}
\hline Yerba compuesta & $\begin{array}{l}\text { Medio de } \\
\text { cultivo }\end{array}$ & $\begin{array}{c}\text { Aspergillus } \\
\text { Sección } \\
\text { Flavi }\end{array}$ & $\begin{array}{c}\text { Aspergillus } \\
\text { Sección } \\
\text { Nigri }\end{array}$ & $\begin{array}{c}\text { Aspergillus } \\
\text { Sección } \\
\text { Circumdati }\end{array}$ & $\begin{array}{c}\text { Aspergillus } \\
\text { Sección } \\
\text { Fumigati }\end{array}$ \\
\hline \multirow{5}{*}{ Marca A } & AA & $10 *$ & 0 & 0 & 0 \\
& PDA & 0 & 10 & 0 & 20 \\
& CZ & 20 & 10 & 0 & 0 \\
& MSA & 10 & 10 & 10 & 0 \\
& MAAS & 10 & 10 & 10 & 0 \\
\hline \multirow{5}{*}{ Marca B } & AA & 0 & 0 & 15 & 5 \\
& PDA & 0 & 0 & 0 & 10 \\
& CZ & 0 & 0 & 0 & 20 \\
& MSA & 10 & 0 & 0 & 10 \\
& MAAS & 10 & 5 & 5 & 0 \\
\hline \multirow{6}{*}{ Marca C } & AA & 5 & 5 & 0 & 0 \\
& PDA & 0 & 15 & 0 & 5 \\
& CZ & 10 & 10 & 10 & 0 \\
& MSA & 0 & 5 & 0 & 5 \\
& MAAS & 10 & 5 & 5 & 0 \\
\hline
\end{tabular}

*Valores expresados como porcentaje de hongos presentes en las muestras analizadas. 
En relación a las incidencias de las diferentes Secciones de Aspergillus spp. entre los diferentes medios analizados, no se observaron diferencias significativas. Esto indica que la diversidad de especies de Aspergillus spp. presentes se podría relacionar al tipo y manejo de muestra y que sería independiente del medio de aislamiento.

\section{CONCLUSIONES}

En las muestras analizadas se indica la presencia de diez géneros de hongos filamentosos, entre ellos cuatro Secciones del género Aspergillus Sección Flavi, Nigri, Circundati y Fumigati. En cuanto a Acremonium sp., Cladosporium sp., Chalara sp., Nigrospora sp., Paecilomyces sp., Torula sp. y Trichoderma sp., es la primera vez que se reporta su presencia en yerba compuesta en Paraguay.

El medio que demuestra mayor eficiencia en el aislamiento de Aspergillus spp. Presente en yerba mate compuesta es el MAAS, medio selectivo para aislamiento de Aspergillus de suelo, puesto que presenta niveles de incidencia mayores de este género en comparación con los otros cuatro medios de cultivo analizados.

\section{REFERENCIAS BIBLIOGRAFICAS}

Abarca, L. 2000. Taxonomìa e identificación de especies implicadas en la aspergilosis nosocomial. Revista Iberoamericana de Micología 17:S79-S84.

Barnett, HL; Hunter, BB. 1986. Illustrated genera of imperfect fungi. 4 ed. New York, USA, Macmillan Publishing. 217 p.

Bezerra da Rocha, ME; Da Chagas Oliveira Freire, F; Feitosa Maia, FE; Florindo Guedes, MI; Rondina, D. 2014. Mycotoxins and their effects on human and animal health. Food Control 36(1):159-165.

Bui-Klimke, TR; Wu, F. 2015. Ochratoxin a and human health risk: a review of the evidence. Critical Reviews in Food Science and Nutrition 55(13):1.

Brasil. 2001. Resolucao RDC ANVISA/MS $\mathrm{N}^{\circ}$ 12: regulamentoténico sobre os padroes microbiológicos para alimentos. Brasilia, BR, Diario oficial da Uniao. 1 p. Secao I.

Brasil. 2002. Resolucao RDC ANVISA/MS N ${ }^{\circ} 302$ : regulamentoténico para fixacao de identidade e qualidade de erva-mate. Brasilia, BR, Diario oficial da Uniao. Brasilia. 67 p. Secao I.

Cabañes, FJ; Abarca, M; Bragulat, MR; Castellà, G. 2007. Especies productoras de micotoxinas In Soriano del Castillo, JM. Micotoxinas en alimentos. ES, Díaz de Santos. p. 30-61.

De Bernardi, LA; Prat Krikum, SD. 2001. Cadena alimentaria de la yerba mate: diagnóstico de la región yerbatera (en línea). Córdoba, AR. Consultado 20 dic. 2015. Disponibe en www.sagpya.mecom.gov.ar/03/ inFusion/diagnòstico/diagnost_YM.htm

Dhingra, OD; Sinclair, JB. 1995. Basic plant pathology methods. 2 ed. US, CRC. 434 p.

Di Rienzo, JA; Casanoves, F; Balzarini, MG; Gonzalez, L; Tablada, M; Robledo, CW. 2011. InfoStat versión 2011 (en línea). Córdoba, AR. Consultado 20 dic. 2015. Disponible en http://www.infostat.com.ar.

Gams, W; Christensen, M; Onions, AHS; Pitt, JI; Samson, RA. 1985. Infrageneric taxa of Aspergillus. In Samson, RA; Pitt, JI. eds. Advances in Penicillium and Aspergillus systematics, New York, US, Plenum. p. 55-64.

Giorni, P; Magan, N; Pietri, A; Battilani, P. 2011. Growth and aflatoxin production of an Italian strain of Aspergillusflavus: influence of ecological factors and nutritional substrates. World Mycotoxin Journal $4: 425-432$.

González, CE; Abrecht, M; Sapper, M; Reckziegel, Y; Fretes, R; Samaniego, R; Paredes, C. 2012. Recuento y caracterización de hongos en yerba mate compuesta con hierbas medicinales. Revista de la Universidad Nacional de Itapúa 6 (6): 44-48.

INTN (Instituto Nacional de Tecnología y Normalización, PY). 1995. Norma Paraguaya 3500193: Yerba mate: especificaciones. 2 ed. Asunción, PY, INTN. 10 p.

IRAM (Instituto Argentino de Normalizaciòn $\mathrm{y}$ Certificaciòn, AR). 2004. Yerba mate canchada y yerba mate elaborada: análisis microbiológicos. AR, IRAM. 1 p.

IRAM (Instituto Argentino de Normalizaciòn $\mathrm{y}$ Certificaciòn, AR). 2007. Norma IRAM 2057: 2007: 
yerba mate canchada y yerba mate elaborada: análsis microbiológicos. AR, IRAM. 1 p.

Jerke, G; Horianski, MA; Salvatierra, KA. 2009. Evaluación de génerosmicotoxigénicos en yerba mate elaborada. Revista de Ciencia y Tecnología 11(12):4145 .

Juan, C; Soriano, JM; Burdaspel, P. 2007. Aflatoxinas del grupo B y G. In Soriano del Castillo, JM. Micotoxinas en alimentos. ES, Díaz de Santos. p. 168-184.

Klich, MA. 2002. Identification of common Aspergillus species. PR, Utrecht, Centraalbureau voor Schimmelcultures. $116 \mathrm{p}$.

Klich, MA; Pitt, JI. 1998. A laboratory guide to common Aspergillus species and their teleomorphs. AU, North Ryde, CSIRO Division of Food Processing. s.p.

Kruskal, WH; Wallis, WA. 1952. Use of ranks in one criterion variance analysis. Journal of the American Statistical Association 260(47):583-621.

López de Cerain, A; Soriano, JM. 2007. Ocratoxina A. In In Soriano del Castillo, JM. Micotoxinas en alimentos. ES, Díaz de Santos. p. 201-222.

Pérez Paiva, J; Piris Morales, L; Ulke Mayans, G; Arias Villalba, M; Mendoza de Arbo, L; Sánchez Bernal, S. 2012. Evaluación del perfil micológico de la yerba mate durante el proceso productivo y producto final en establecimientos yerbateros. Revista de Salud Pública del Paraguay 3(1):8-13.

REDIEX (Red de Inversiones y Exportaciones, PY). 2015. Yerbateros buscan aumentar el cultivo para más producción (en línea). Consultado 02 jun. 2015. Disponible en http://www.rediex.gov.py/yerbaterosbuscan-aumentar-el-cultivo-para-mas-produccionnot431
Robert, V; Vu, V; Ben Hadj Amor, A; Van de Wiele, N; Brouwer, C; Jabas, B; Szoke, S; Dridi, A; Triki, M; Ben Daoud,S; Chouchen, O; Vaas, L; de Cock, A; Stalpers, JA; Stalpers, D; Verkley, GJM; Groenewald, M; Borges dos Santos, F; Stegehuis, G; Li, W; Wu, L; Zhang, R; Ma, J; Zhou, M; Pérez Gorjón, S; Eurwilaichitr, L; Ingsriswang, S; Hansen, K; Schoch, C; Robbertse, B; Irinyi, L; Meyer, W; Cardinali, G; Hawksworth, DL; Taylor, JW; Crous, PW. 2013. MycoBank gearing up for new horizons. IMA Fungus 4(2):371-379.

Samson, RA; Visagie, CM; Houbraken, J; Hong, SB; Hubka, V; Klaassen, CHW; Perrone' G; Seifert, KA; Susca, A; Tanney, JB; Varga, J; Kocsubé, S; Szigeti, G; Yaguchi, T; Frisvad, JC. 2014. Phylogeny, identification and nomenclature of the genus Aspergillus. Studies in Mycology 78:141-173.

Sanchis, V; Marin, S; Ramos, A. 2007. Factores determinantes en la producción de micotoxinas. In Soriano del Castillo, JM. Micotoxinas en alimentos. ES, Díaz de Santos. p. 63-89.

Soriano, JM. 2007. Introducción. Micotoxinas en alimentos. ES, Díaz de Santos. 393 p.

Shapiro, SS; Wilk, MB. 1965. An analysis of variance test for normality (complete samples). Biometrika 52:591-592.

Wu, F; Groopman, JD; Petska, J. 2014. Public health impacts of foodborne mycotoxins. Annual Review of Food Science and Technology 5:351-372. 